\title{
Measurement Method and Result for Frequency Spectrum Sharing in Cognitive Radio
}

\author{
Osamu Takyu ${ }^{*}$, Shunta Horie ${ }^{\dagger}$, Hasan Rajib Imam ${ }^{\ddagger}$, Masahiro Furuichi ${ }^{\dagger}$, Takamasa Kimura ${ }^{\ddagger}$, \\ Takeo Fujii $\ddagger$, and Yohtaro Umeda $\dagger$ \\ * Shinshu University, Japan \\ $\dagger$ Tokyo University of Science, Japan \\ †The University of Electro-Communications, Japan \\ E-mail: takyu@rs.noda.tus.ac.jp
}

\begin{abstract}
In this paper, we construct two measurement systems for specifying the channel occupancy ratio. Channel occupancy ratio is useful for cognitive radio because it is estimation for finding the vacant channel. We consider two type of channel occupancy. In the average channel occupancy, we detect the signal from low frequency (LF) to Ultra High Frequency (UHF) for long terms. As a result, the average channel occupancy for each frequency channel is specified. In addition, we estimate the instantaneous channel occupancy by real time spectrum analyzer. We also construct the combining system between measurement and simulation and thus various signal detectors are available for the constructed system. From the measurement result, we analyze the MAC level channel accessing from the detected result in some cases.
\end{abstract}

\section{INTRODUCTION}

In mobile wireless system, exhausting frequency spectrum is serious problem. For recovering the problem, the cognitive radio concept is attracting much attention[1]. In cognitive radio, the independent mobile terminal finds the vacant frequency spectrum which no system uses for wireless communication and then it constructs wireless communication in the vacant frequency spectrum without the interference to any other system. Since the cognitive radio aggressively uses the vacant frequency spectrum, the efficiency of frequency spectrum usage is much improved.

For finding the vacant frequency channel, the time periodical information of channel occupancy is useful, where it means how much time and how frequently the other system occupies the frequency channel[2]. We can consider two types of channel occupancy. First one is instantaneous channel occupancy and is useful for avoiding the simultaneous accessing of multiple users. Second one is long term channel occupancy and becomes estimation for the other terminal to find the vacant channel. We note that the period of channel occupancy depends on the application of the system. In the regulation of frequency spectrum, each frequency band is used for the specified application, such as ultra high frequency (UHF) is used for TV service and $2.4 \mathrm{GHz}$ is used for industry science and medical (ISM) band. Since the time period of channel occupancy is different for each application, the measurement experiment of channel occupancy for each frequency band is necessary.

In this paper, the measurement system for field experiment is developed, where it is suitable for cognitive radio system. For specifying the long term channel occupancy of each frequency band, the measurement system of channel occupancy
TABLE I

EQUIPMENTS USED IN THE EXPERIMENT

\begin{tabular}{|c|c|}
\hline Equipment & Detail \\
\hline \hline Antenna & $\begin{array}{c}\text { DIAMOND ANTENNA } \\
\text { Sup erDiscon D3000N } \\
(25 \mathrm{MHz} \sim 3 \mathrm{GHz})\end{array}$ \\
\hline $\begin{array}{c}\text { Spectrum } \\
\text { Analyzer }\end{array}$ & R\&S FSC6 (9kHz $6 \mathrm{GHz})$ \\
\hline PC & PanasonicLet's Note CF-W8 \\
\hline Cable & Antenna Cable, Gap Line Flat Cable \\
\hline Connector & $\begin{array}{c}\text { Antenna-Spectrum Analyzer } \\
\text { Connector }\end{array}$ \\
\hline
\end{tabular}

for long term, some hours or some days period, is constructed. We show the results of long term channel occupancy from the measurement of field experiment and clarify the dependency between the channel occupancy ratio and frequency channel.

In addition, we also evaluate the instantaneous channel occupancy, such as $\mu \mathrm{sec}$ order. We also develop the measurement system composed of measurement part and simulation one. In measurement part, the electrical power versus time is used. The evaluated results are put into a computer by off line not real time. As a result, the relationship between the electrical intensity and time can be simulated in computer. Since in the constructed measurement system, the signal processing based on computer simulation is available, various signal detectors is applied to the detected signal. In this paper, we consider two methods, energy detection and cooperative detection and then the detection accuracies of them are evaluated. From the field experiment, we clarify the accuracy of the evaluated instantaneous channel occupancy.

\section{WIRELESS COMMUNICATION ENVIRONMENT AND PREDICTION CONSIDERING TRAFFIC}

As the wireless devices are increasing to a great deal, the issue of the shortage of the spectrum band is becoming a big problem. To solve this issue, Cognitive radio is attaining a great attention for effective spectrum sharing for both the primary user (PU) and the secondary user. It is necessary to realize how PU is using the spectrum band, which has the priority in this band. Therefore, in order to realize the current usage of spectrum, the received signal power is measured using the spectrum analyzer in different frequency band. The occupancy of the PU is measured based on these data.

\section{A. Experiment configuration}

As shown in Fig. 1 and Fig. 2, an antenna is installed on the roof of a building at The University of Electro- 


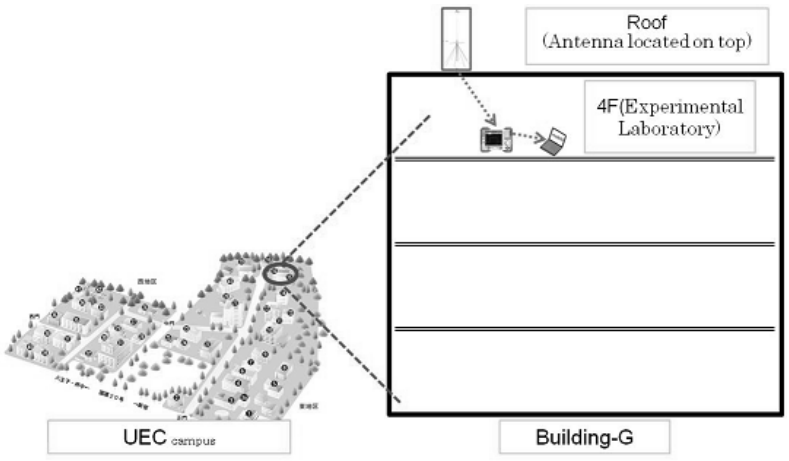

(a) Measurement location

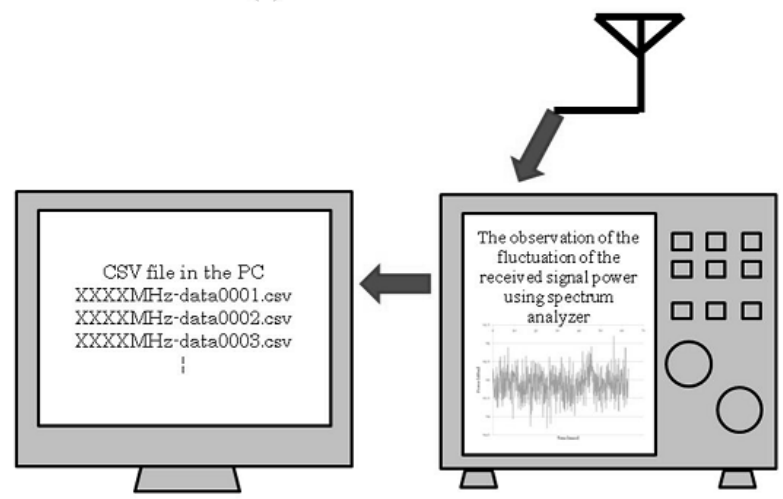

(b) Configuration of the equipment

Fig. 1. Environment of measurement

Communications and the radio wave of the Choufu-city Choufu-Gaoka is measured, where table I shows the specifications of measuring equipments. Choufu-city is located in the western suburban area of Tokyo Metropolis, Japan. The building considered as the measurement location in this case is a four-storied building with the area of 541 square meter of land. The received signal power of the spectrum is measured by the spectrum analyzer which is set up in indoor. The measured data is stored in the computer in CSV file format. The mode of the environment is set into 'Zero Span' and only the data of one spectrum band is achieved in one observation. The equipments and the antenna that are used in the experiment are showed in Fig. 3.

\section{B. Evaluation of threshold value to measure channel occu- pancy}

In order to realize whether the spectrum is used by the PU or not, the evaluation of the threshold value is necessary. If the instantaneous received signal power exceeds the threshold value, the band is considered to be occupied. As shown in Fig. 3 , it is difficult to understand the occupancy of the PU in the spectrum band from the observation of the fluctuation of the received signal power only. Hence, in this measurement, the threshold signal power is defined to understand whether PU is using the spectrum band or not.

The degree of the internal noise and the external noise of the equipment is varied with the frequency spectrum band. Therefore, the threshold power is also expected to be varied for different band. The threshold value, $T$ to evaluate the

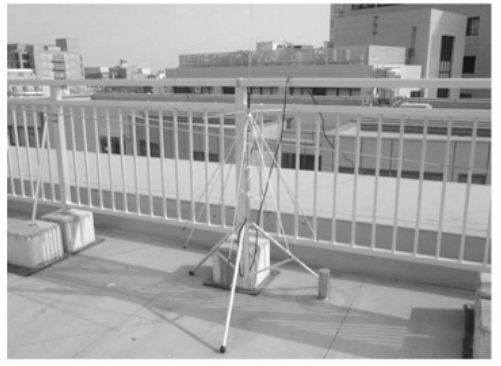

(a) Antenna

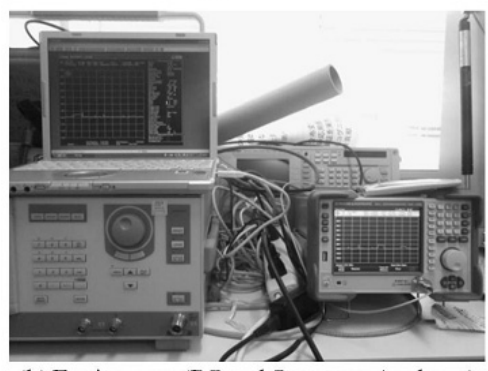

(b) Equipments (PC and Spectrum Analyzer)

Fig. 2. Equipments used in the experiment

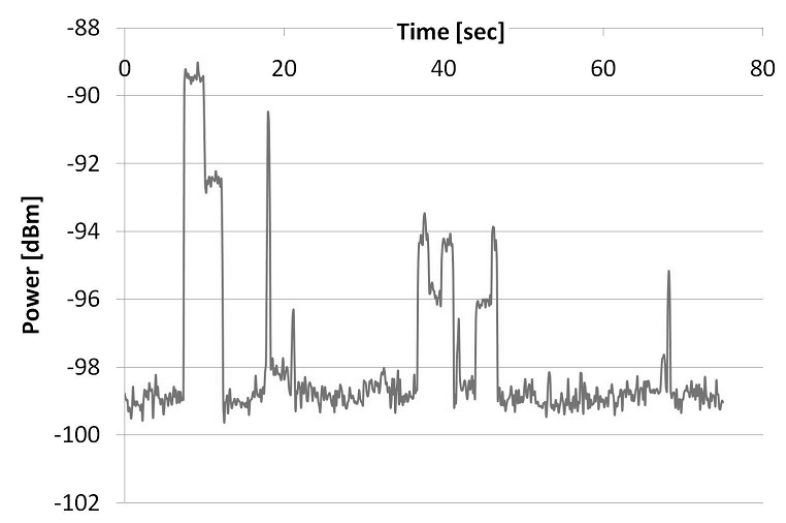

Fig. 3. The received signal power at $2.4 \mathrm{GHz}$ band

occupancy rate is shown in equation (1). The external noise $n_{0}$ is evaluated by finding the spectrum band where there is no radio wave signal, and by evaluating the average of the measured results observed at Max Hold for a certain time in that band. The internal noise $n_{i}$ is evaluated by putting the receiver cable inside an anechoic chamber box and by evaluating the average of the measured results observed at Min Hold in the same frequency band. Finally, the threshold power is calculated from the average of the internal and the external noise.

If the number of the measured data that exceeds the threshold value is $x_{u}$, and the number of the data that does not exceed the threshold value is $x_{d}$, therefore, the spectrum band occupancy rate $O$ can be determined from equation (2).

$$
\begin{array}{r}
T=\operatorname{AVERAGE}\left(n_{o}+n_{i}\right) \\
O=\frac{x_{u}}{x_{u}+x_{d}}
\end{array}
$$


TABLE II

EXPERIMENTALRESULT

\begin{tabular}{|c|c|c|c|}
\hline $\begin{array}{c}\text { Measured Band } \\
\text { Frequency[MHz] }\end{array}$ & The Kind of Primary System & $\begin{array}{c}\text { Threshold Power for } \\
\text { Duringuse or Not[dBm] }\end{array}$ & Occupancy[\%] \\
\hline \hline 60.0000 & $\begin{array}{c}\text { Accident Prev ention for government/Local Public } \\
\text { Agency/Municipality-Fixing System }\end{array}$ & -101.43 & 1.73 \\
\hline 800.0000 & Radio Microphone/Type-A/AL-15ch & -115.94 & 2.99 \\
\hline 810.0500 & $\begin{array}{c}\text { Cell-phone/FDC·PDC (Personal Digital Cellular)- } \\
\text { method/BaseStation }\end{array}$ & -110.69 & 17.71 \\
\hline 885.0125 & Airport Radio Service/Base Station/MCA-method & -114.15 & 44.22 \\
\hline 962.0000 & $\begin{array}{c}\text { Aeronautical Radio/Marine Navigation } \\
\text { /DME above Grade/Tactical Air Navigation }\end{array}$ & -112.32 & 39.10 \\
\hline 1525.0025 & Inmarsat Satellite/Downlink & -119.00 & 36.08 \\
\hline 1575.2420 & Global Positioning System/ForCivilian use & -112.32 & 32.92 \\
\hline 1673.0000 & Weather SupportCenter/Ground Radar/Radiosonde & -111.42 & 2.12 \\
\hline 2412.0000 & 2400MHz Ham Radio/Wireless LAN & -98.62 & 32.65 \\
\hline
\end{tabular}

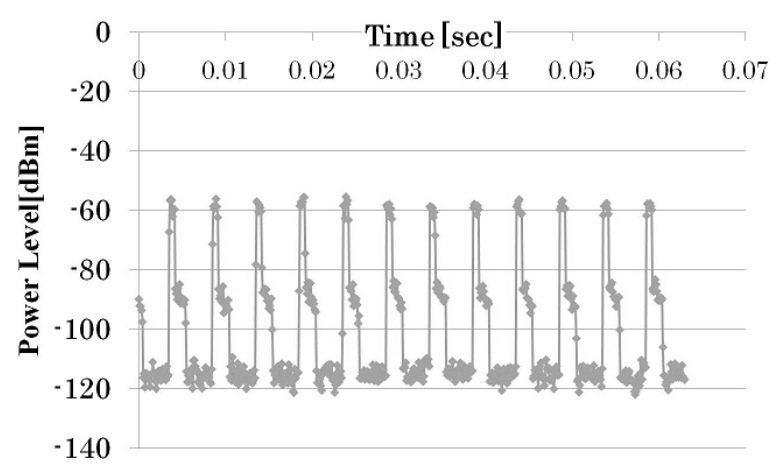

Fig. 4. The periodicity in PU transmission (1900.55MHz: PHS/ Transmission Channel/ All over the country)

\section{Observed data}

In Table II, the occupancy rate of different spectrum band is shown. Each of the spectrum bands is measured for 4.5 hours in this case. The data is measured one time in every 4 seconds, and each time, the data of 630 points is achieved in every $0.1[\mathrm{msec}]$. The results shown in Table II and the fluctuation of the received signal power shown in Fig. 3 do not show the periodicity. For comparison, the fluctuated received signal power which shows the periodicity is shown in Fig. 4. In this case, the SU detects the periodicity of the primary received signal power, and can share the PU spectrum band. However, as shown in Table 2, most of the PU does not make the periodic transmission only. For the secondary use of the primary spectrum band, it is necessary not only to detect the PU transmission but also to make the proper resource control in spectrum sharing.

\section{Conclusion}

As the primary band occupancy rate shown in table 2 is less than $50 \%$, the spectrum band can be considered to be used secondarily if the threshold values calculated are collected. However, the measured data are confined to a certain time and date. The measured location is also confined to the Chofu-city.

\section{E. Works to do}

The above experiment was conducted for 4.5 hours at a certain location and for a specific time. As a next step of this experiment, we want to make the measurements of the radio

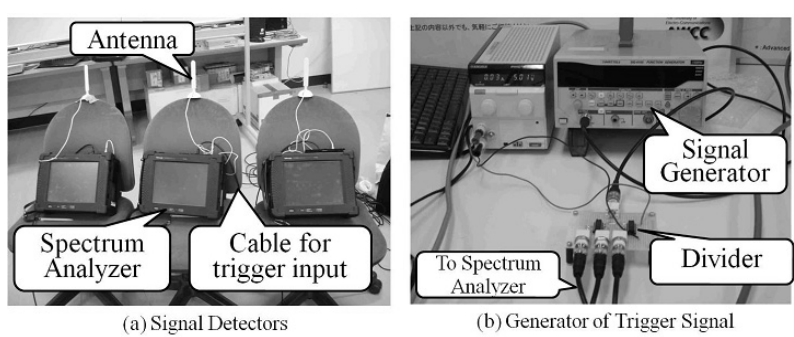

Fig. 5. Equipments of Constructed Measurement System

wave information for a longer time. We are also thinking of changing the location and time of the measurement and finally make the comparison amo

\section{Small Scale Measurement}

\section{A. Overview of Measurement}

Fig. 5 shows the equipments of constructed measuring system. There are three equipments, first one is real time spectrum analyzer (SA 2600, Techtolonics Products), second one is omni-directional antenna (CG-WLAANT011, Corega Product) and final one is signal generator for measurement's trigger. In SA2600, Amplitude Versus Time, which is one of software for analyzing the detected signal, can memorize the band limited signal for constant time duration. This application can resolve detected signal into In-phase (I) component and Quadrature phase $(\mathrm{Q})$ one. The minimum time domain resolution is strictly determined by the detection bandwidth. As the detection bandwidth becomes large, the sampling rate becomes larger and thus the time resolution of detected signal becomes finer [3]. Since the size of memory is constant, the time duration of memorizing the detected signal should be small when the sampling rate becomes larger.

SA2600 is equipped with the external trigger and thus the timing of measurement can be controlled by external trigger signal. The external trigger of it is composed of transistortransistor logic (TTL) so the controller of measurement is based on logic. When the external trigger is used, multiple detectors can be controlled by common trigger signal. As a result, the simultaneous measurements system can be constructed. Fig. 5 shows the system overview of simultaneous measurement system. This system is composed of three parts. First one is multiple detectors (real time spectrum 


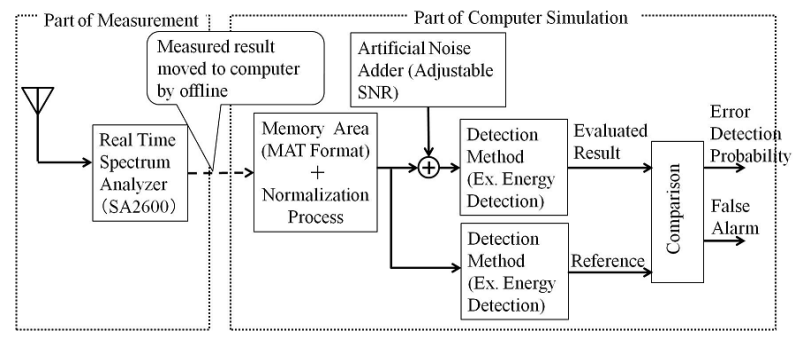

Fig. 6. System Diagram of Combined System between Measurement and Simulator

analyzer(SA2600)), second one is signal generator for trigger, and third one is the divider for constructing common trigger signal. After signal generator generates the rectangle signal, the generated signal is put into the one-to-multiple divider, where the divider is composed of three line drivers (Texas Instruments, SN74S140N) . After that, each generated signal is put into the trigger input of SA 2600. We use "rising edge" so when the voltage of signal is changed from low level to high one, SA2600 starts to detect the signal. Once SA2600 detects the signal, the detected acquisition is stopped due to one shot function. In the constructed measurement system, some detectors, SA2600, are used and then can be controlled by single trigger signal. If the signal arrival or some error function is negligible, all the detectors acquire the electrical wave signal, simultaneously. Since the detections of the electrical wave signal can be detected in the multiple different locations, simultaneously, the electrical wave signal can be resolved in terms of not only time domain but also space domain.

\section{B. Application of Constructed Measurement System part-1: Evaluating the accuracy of signal detector}

It is one of the applications for constructed measurement system to evaluate the accuracy of signal detector. In the constructed measurement, we can deal with the I-phase and Q-phase components of detected signal, separately. Therefore, the instantaneous sampled signal is transformed into complex signal and thus can be applied to the signal processing including the signal detector. Note that coherent detection needs the phase synchronization but it is not available owing to nosynchronization. In packet switching, such as wireless LAN, the signal exciting depends on the packet exciting. Therefore, if the packet exciting is exactly known, the evaluation of detection probability based on the exciting of signal as well as that of false alarm based on the vacant of signal can be performed.

The signal is acquired under the constant signal to noise power ratio (SNR) but for evaluating the robustness of noise component, it is desirable that SNR is adjustable. In this paper, we consider the combined system between field measurement and simulation one. Fig. 6 shows the block diagram of it. In field measurement part of the combined system, the acquired data is formatted by MATLAB data formatting (MAT) and then it is moved from SA 2600 to Personal Computer by USB or other data interface. We note that the measurement result is not moved to computer in real time but in off-line. For moving the evaluated result into computer simulation, the USB memory or other network interface is available.

For detecting packet emitting, one of detectors is set near the test subject of transmitter. It is referred to as reference detector. The SNR of detected signal is so high that the noise component can be considered as negligible. Therefore, in reference detector, the time transition of emitted signal can be evaluated under high accuracy. As a result, the packet emitting is almost perfectly evaluated. Even if the other detector is under the low SNR, the packet exciting can be specified by the packet acquisition from the reference detector.

Next, for controlling the SNR of acquired data, we consider the quasi noise components are added into this data. For deciding the power level of quasi noise components, we firstly evaluate the power of desired signal. Since we can specify the packet signal due to reference detector, we collect the power of packet signal. After that, the average power per one instantaneous sample is evaluated and thus the required power of quasi noise component for achieving the desired SNR can be calculated. After the quasi noise components are added into the acquired data signal, the SNR is adjusted. From this manner, we can control the SNR of acquired data signal.

We explain how to evaluate the accuracy of detection method in the constructed measurement system. As we show Fig. 6, we use the two data signal, one is original data and the other one is the data signal into which the quasi noise component is added. We apply both signals to the process of detection method. If the results from both signals are the same, the result from the signal into which the quasi noise component is added is true, otherwise it is false. If the packet is in the data signal and the result is false, we consider this is false detection. If the packet is not in it and the result is false, we consider is false alarm. As we explain, the packet exciting can be known from the reference detector. From this manner, we can evaluate the detection probability and false alarm probability of the target detection method.

\section{Application of Constructed Measurement System part-2: Analyzing MAC level Wireless Accessing}

Since the constructed measurement can detect the microsec order signal detection, the data packet and ACK packet are resolvable. From the acquired data from the constructed measurement, the accessing ratio of channel can be evaluated. In addition, in the constructed measurement, the measurement in multiple locations can be performed. Therefore, the acquired data can be resolved for the time domain and the space one. Therefore, the relationship between the wireless electric propagation and the MAC level wireless accessing should be clarified.

\section{Evaluation Results}

In this section, we show the measurement result in the indoor experiment. Table III shows the simulation parameters. Fig. 7 shows the field map of measurement indoor environment. We consider three detectors, where detectors are referred 
TABLE III

SYSTEM PARAMETERS

\begin{tabular}{|l|l|}
\hline Target System & IEEE $802.11 \mathrm{a}$ \\
\hline Detector & \\
Center Frequency & $5.1281290 \mathrm{GHz}$ \\
Frequency Bandwidth & $200 \mathrm{kHz}$ \\
Sampling Rate & $280 \mathrm{kbps}$ \\
Duration Time & $357.139286 \mathrm{msec}$ \\
Reference Level & $-30 \mathrm{dBm}$ \\
\hline
\end{tabular}

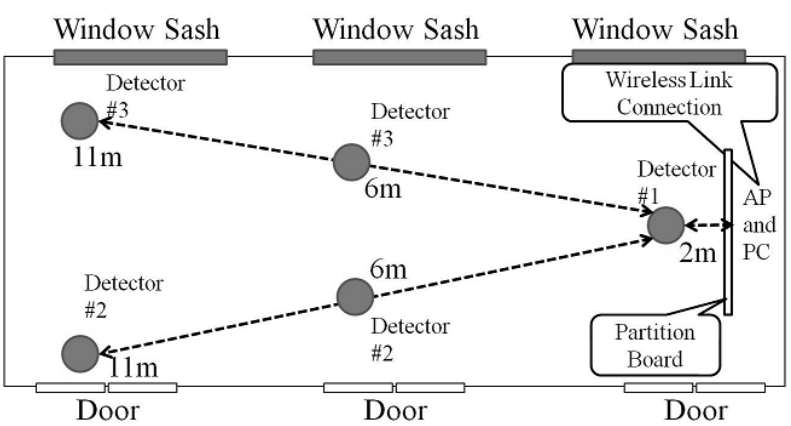

Fig. 7. Field Map of Indoor Environment

to as "\#1","\#2", and "\#3", respectively. The role of "\#1" is reference detector for detecting the packet exciting.

Fig. 8 shows the performance between SNR and miss detection probability where we use the energy detector [4]. Since the simultaneous detection by two detectors, "\#2" and "\#3",is available, we can also consider cooperative detection [5] between " $\# 2$ " and " $\# 3$ ". The time duration of charging is one sample. As the false alarm is 0.1 for all the performance, the decision line can be decided. From this figure, when the distance of " $\# 2$ " and " $\# 3$ " from " $\# 1$ " is $6 \mathrm{~m}$, the cooperative detection achieves $2 \mathrm{~dB}$ smaller SNR than each single detector. This is because the number of samples becomes twice and thus the energy of detected signal becomes almost twice. However, when the distance of two detectors is $11 \mathrm{~m}$, the performance with " $\# 2$ " is much different from the performance with " $\# 3$ ". We guess that since the distance between " $\# 2$ " and "\#3" becomes large, the wireless propagation of them are different each other. The detection accuracy with cooperation is slightly better than that with "\#2". Even if the part of result is worse, the cooperation makes the detection accuracy improved.

Fig. 9 shows the evaluation result of time versus amplitude, where we use the result measured by "\#1". For clarifying the packet exciting, we set about $2 \times 10^{-5}$ as decision line and then if the detected sample is larger than decision line, it is 1 otherwise 0 . From this figure, we can see that the duration between the long duration of one and the short one is 6 samples, where 6 samples are almost equal to $21.4 \mu \mathrm{sec}$ and thus is almost equal to SIFS time duration [6]. The accuracy of detecting ACK packet is not large because some ACK packets are missing. Therefore, the measurement method of detecting data packet and ACK packet, separately is necessary. For example, we use two detectors and then one detector is near the transmitter and the other one is near the receiver. As a result, the signal power of Data is large at transmitter and that of ACK packet is large at receiver, respectively. This is one of the methods for separately detection.

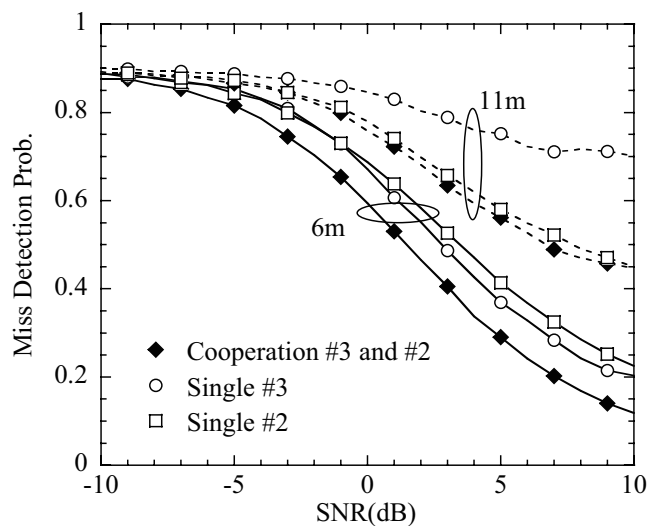

Fig. 8. Performance between Miss Detection Prob. and SNR

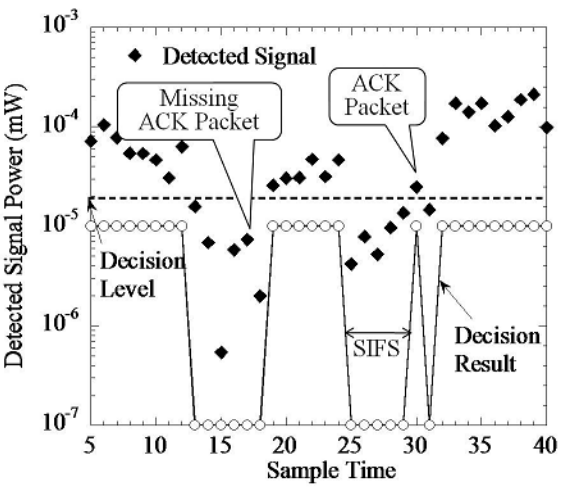

Fig. 9. Snapshot between sample time and instantaneous power

\section{CONCLUSION}

In this paper, we consider two measurements system and then show the some examples of data. Each result still has some problems and thus recovering them is important future work.

\section{ACKNOWLEDGEMENT}

A part of this research project are sponsored by Ministry of Internal Affairs and Communications in Japan as a project name of Strategic Information and Communications R\&D Promotion Programme (SCOPE 092103009) and KAKENHI (20760248).

\section{REFERENCES}

[1] S. Haykin, "Cognitive radio: brain-empowered wireless communications," IEEE Journal on Selected Area in Communications, vol. 23, no. 2, pp. $201-220$, Feb. 2005

[2] S. Tanaka, T. Fujii, " Development of Intelligent MAC Protocol on Autonomous Distributed Control for Cognitive Radio," Proc. TriSAI2009, Tokyo, Japan, Oct. 2009.

[3] http://www2.tek.com/cmsreplive/psrep/14795/37W_23237_3_ 2011.03.04.12.55.16_14795_EN.pdf

[4] Steven M. Kay, Fundamentals of Statistical Signal Processing Volume II Detection Theory, Prentice-Hall PTR, 1998

[5] H. Uchiyama, et al, "Study on Soft Decision Based Cooperative Sensing for Cognitive Radio Networks," IEICE Trans. Commun. vol. E91-B, no.1, pp.95-101, January 2008

[6] ISO/IEC8802-11 ANSI/IEEE Std 802.11, "Information technology, Part 11 : Wireless LAN Medium Access Control (MAC) and Physical Layer (PHY) specifications”, 1999 